\title{
A NOVEL APPROACH FOR ENERGY EFFICIENT HIERARCHY BASED ROUTING IN SENSOR NETWORKS
}

\author{
S.Karthikeyan ${ }^{1}$ and S.Jayashri ${ }^{2}$ \\ ${ }^{1}$ Research scholar, Sathyabma University, chennai, India \\ karthijoyl@gmail.com \\ ${ }^{2}$ Adhiparasakthi engineering college, Melmaruvathur, Tamil Nadu, India \\ jayaravi2010@gmail.com
}

\begin{abstract}
Wireless sensor network (WSN) is the collection of many micro-sensor nodes, connecting each other by a wireless medium. WSN exhibits different approaches to provide reliable sensing of the environment, detecting and reporting events. In this paper, we have proposed an algorithm for hierarchy based protocols of wireless sensor networks, which consist of two groups of sensor nodes in a single cluster node. Each cluster consists of a three cluster head. The event driven data sensing mechanism is used in this paper and this sensed data is transmitted to the master section head. Hence efficient way of data transmission is possible with larger group of nodes. In this approach, using hierarchy based protocols; the lifetime of the sensor network is increased.
\end{abstract}

\section{KEYWORDS}

Sensor Nodes, lifetime, event driven, master section head.

\section{INTRODUCTION}

In recent period, efficient design and implementation of wireless sensor networks had become a hot area of research. It is due to the vast potential of sensor networks for enabling applications that will be able to connect the physical world to the virtual world. By the use of networking a huge number of tiny sensor nodes, it is being made to obtain data about physical phenomena which was in fact difficult or almost impossible to obtain in more conventional ways. In future, as micro-fabrication technology will get advanced which will ultimately allow the cost of manufacturing sensor nodes to fall, which is expected to increase deployments of wireless sensor networks, with the networks growing rapidly to large number of nodes, for e.g., thousands[1]. The data transmission consumes more energy in WSN, when compared to data processing. The data aggregation will balance the energy consumption of each node, so that the network's lifetime is increased [2],[3].

A large number of potential applications for such a large scale wireless sensor networks are existing in a variety of fields which include medical monitoring, environmental monitoring,

Sundarapandian et al. (Eds): CoNeCo,WiMo, NLP, CRYPSIS, ICAIT, ICDIP, ITCSE, CS \& IT 07, pp. 491-500, 2012. () CS \& IT-CSCP 2012

DOI : $10.5121 /$ csit.2012.2448 
surveillance, security, military operations and industrial machine monitoring. For the better understanding as to why traditional protocols are not the best suited for these types of sensor network applications, in the remainder of this section we will be categorizing the unique features of sensor networks and the performance metrics with which the protocols for the sensor networks must be evaluated.

\subsection{Features of WSN}

It should be pointed that sensor network will share some commonalities with general ad hoc networks. Thus protocol design [4] which is used for sensing networks must account for the properties of ad-hoc networks, including the following.

- Life time constraints will be imposed by limited energy supplies of the nodes in the network. An unreliable communication due to a wireless medium.

- The need for self-configuration, which requires either little or no human intervention.

○ However several unique features will exist in wireless sensor network that doesn't even exist in general ad hoc networks. These features will have new challenges and will require modification of designs for traditional ad hoc networks.

- While traditional ad hoc networks will consist of the network size in the order of 10's, sensor networks are expected of the size 1000s.

- The sensor nodes are typically immobile, which clearly means that the mechanism used in traditional ad-hoc network protocols to deal with mobility may be unnecessary and also may look overweight.

- As nodes can be deployed in harsh environmental conditions, unexpected node failure is very common.

○ Sensor nodes may be much smaller then nodes used in traditional ad hoc networks (e.g., PDAs, laptop computers), consisting smaller batteries which leads to shorter life time, less computational power and also less memory.

There are also additional services such as location information which may be required in wireless sensor networks. While nodes in the traditional ad hoc networks compete for resources such as bandwidth, nodes in a sensor network can be expected to behave more cooperatively, as they are trying to accomplish a similar universal goal, which are typically related to maintaining an application-level quality of service (QOS), or fidelity. Communication is actually data datacentric rather then address-centric, which means that routed data may be aggregated or compressed or prioritized or dropped depending on the description of the data. Communication in the sensor networks typically takes place in the form of very short packets, which means that the relative overhead imposed at different network layer had become much more important. 


\section{TAXONOMY FOR ROUTING PROTOCOLS}

Classification of routing protocols for network structure are

\subsection{Flat-based routing}

In the flat based routing, feature of unique global identifier can not be provided for each node because of large number of nodes. Deployed nodes in the networks are assigned with equal roles. Data-centric routing, where queries are given by the destination node to the particular region of the nodes. Data delivered after a delay period from the region of that particular sensed node. Data request is based on queries, properties of the data is specifically necessary for this attribute based naming. For e.g., SPIN, Rumour routing, DD and Gradient based routing (GBR).

\subsection{Hierarchical-based routing (Cluster-based routing)}

Different roles can be played by the sensor nodes in the networks and the protocol is based on the cluster creation. Cluster creation and specific task assignment to cluster head contributes the overall system energy efficiency, scalability, and lifetime of the network. Hierarchical routing performs data fusion and aggregation which decreases the number of information message transmitted to the destination node. It gives the energy efficient way of reducing the energy consumption in the cluster.

In addition to this different task of the sensor nodes with different characteristics of data transmission is performed.

for e.g., Low Energy Adaptive Clustering Hierarchy (LEACH), Power-Efficient Gathering in Sensor Information Systems (PEGASIS), Threshold-Sensitive Energy Efficient Protocols (TEEN).

\subsection{Location-based routing}

Location based addressing of the nodes is formed in this routing protocol. Distance between the node A and the neighbour is estimated by calculating the signal strength or by GPS receivers. For e.g., Geographic Adaptive Fidelity (GAF), Geographic and Energy Aware Routing (GEAR).

\section{HIERARCHY BASED PROTOCOL}

\subsection{LEACH}

LEACH is cluster-based protocol. It has distributed cluster formation. In LEACH at first random selection of the cluster head from the group of distributed nodes. The role of cluster head is rotated to evenly distributed energy of the sensor nodes in the networks. LEACH the cluster head $(\mathrm{CH})$ compress the information packet received from different nodes within the same cluster and the aggregated data packet are transmitted to the base station which reduces the size of the information to be transmitted to the base station. This protocol uses the TDMA/CDMA medium access control (MAC) which reduces collision in the inter-cluster and Intra-cluster data transmission. Centralized data collection is done in periodical basis. In this protocol, constant monitoring of the sensor nodes in the networks. User does not need all the data instantaneously. 
So, periodically data transmission will drain the limited energy of the sensor nodes in the network. Unnecessary data transmission is avoided to save energy in the sensor nodes. After a period of time, rotation of the cluster head is randomized. So that energy distributed in the sensor nodes will be event after certain period of time. LEACH is operated into two phases, the setup phase and the steady state phase. In the setup phase, organized clusters and Cluster head selection takes place. In the steady state phase, the actual data is transfer to the base station. To minimize the overhead, the steady state phase duration is longer than the duration of the setup phase. In the setup phase, a predetermined fraction of nodes, p, elect themselves as CHs as follows. Sensor node with the random number ( $r$ ) is chosen between o and1. Compared random number is less than the threshold value, $\mathrm{T}$ (n), for the current round the node becomes a cluster-head. The threshold value is calculated based on the desired percentage to become a cluster head, current round and nodes that has not appeared as a cluster-head in the last $(1 / \mathrm{p})$ rounds, which is denoted by $\mathrm{G}$. Where $\mathrm{G}$ is the group of nodes involved in the cluster head selection process. After $\mathrm{CH}$ selection, $\mathrm{CH}$ broadcast an advertisement message as a new cluster-heads to the group of the sensor nodes in the network [6]. Non-cluster head receives the advertisement message and from there it decides which cluster they want to join. Based on the signal strength of the advertisement the nodes grouped as a cluster. Member allocation takes place that the non-cluster nodes inform to the $\mathrm{CH}$ that they will be a part of this cluster. Messages from different nodes are received by the $\mathrm{CH}$ that it would like to be a part of the cluster, based on the number of nodes in the cluster, TDMA schedule is created by the cluster-head nodes and each node is assigned with a time slot by which it can transmit.

This schedule is being broadcasted to each and every node present in the cluster. The senser nodes are able to sense and transmit the data to the cluster heads during the steady state phase. The cluster head node receives all the data and then it will aggregate all the data before sending the data to the base station. After a definite period of time, which will be called as priori the network will go back to the setup phase and then it will go for another round of selecting new $\mathrm{CH}$. Cluster will use different CDMA codes to communicate, this is done to reduce interference from nodes which are belonging to other cluster. Network lifetime will be increased by leach, but there will be a number of issues for the assumptions used for this protocol.

The main work of LEACH is to watch that whether all nodes can transmit with sufficient power to reach the BS if it is required to. With this each node will have computational power for supporting different MAC protocols. For this networks are not allowed to be deployed in large regions. It predicts that nodes must always consists data to send \& the nodes which are located nearby will have correlated data. It is not necessary that the number of predetermined $\mathrm{CHs}$ (p) should be uniformly distributed throughout the network. Hence there is a probability that the elected CHs could be concentrated in any one part of the network. Therefore there is a possibility that that some nodes will not be having any $\mathrm{CH}$ 's in their vicinity. Moreover, the concept of dynamic clustering brings an additional concept for eg., head changes, advertisements etc., which may reduce the gain in the energy consumption. At last the protocols assure that all nodes should begin with the same amount of energy capacity in each of the election round. For this it is assumed that $\mathrm{CH}$ will be consuming almost the same amount of energy for each node. The protocol should extend to account for non uniform energy nodes, i.e., by using energy-based threshold. 


\subsection{TEEN and APTEEN}

TEEN (Threshold-sensitive Energy Efficient sensor Network) and APTEEN (Adaptive Periodic Threshold-sensitive Energy Efficient sensor Network protocol) are the two hierarchical routing protocols which are being proposed for time-critical applications. In case of teens, the medium is being sensed by the sensor nodes, whereas data transmission is done very rarely. A cluster head is used to send its members a hard threshold which results as the threshold value of the sensed attribute and a soft threshold, which is actually a differential change in the value of sensed attribute that is used to trigger the node to switch on its transmitter and it transmit's. Now the hard threshold get activated and it tries to reduce the number of transmission by allowing the nodes to transmit only sensed attribute[4] comes under the range of interest. The soft threshold in result reduces the number of transmission otherwise it may happen when there is a little or no change in sensed attribute. When the value of soft threshold is small it gives a more perfect picture of the network, at the expense of increased energy consumption. In such cases, the user is able to control the trade-off between energy efficiency and data accuracy. When cluster-heads are needed to be changed, new set of values are broadcasted for the above parameters.

The ultimate drawback of the scheme is that, if any case the threshold is not received, the nodes can never communicate and the user will not be able to get any sort of data from the network. The nodes will be sensing their environment continuously. When for the first time a parameter from attribute set reaches its hard threshold value, the node will switch its transmitter in on state and then it will send the sensed data. The sensed value store in an internal variable known as sensed value (sv). The nodes are being able to transmit data in the period of current cluster only if the following conditions are satisfied. The conditions are:-

1) The current of the sensed attribute must be greater then the hard threshold.

2) The current value of the sensed attribute must differ from SV by an amount equal to or greater then the soft threshold.

The important features of TEEN include its suitability for the time critical sensing applications. Also, as message transmission will consume more energy then data sensing, so the amount of energy consumption in this scheme is less then the proactive networks. The soft threshold can also be varied. For every cluster time change, a set of fresh parameters are broadcasted and so, the user can change them as it is required.

The periodicity and threshold values are being changed by APTEEN which is a hybrid protocol \& are being used in the TEEN protocol as per the user needs \& type of the application[6]. The following parameters are being broadcasted by the cluster heads in the APTEEN.

1. ATTRIBUTE: - A set of physical parameters which is obtained by the user at his self interest.

2. THRESHOLDS: - Hard threshold (HT) and soft threshold (ST) are the two parameters of the threshold.

3. SCHEDULE: - This is a TDMA schedule, which assigns slots to each node.

4. COUNT TIME (CT):- This is the maximum time period obtained between two successive reports which is being sent by a node.

The node will sense the environment continuously and only those nodes which sense a data value at or beyond the hard threshold will transmit. Once a node is able to sense a value beyond HT, it will transmit data only if the values of that attribute changes by an amount that is equal to greater 
then ST. If in some case a node does not send data for a particular time period which is equal to count time, it is being forced to sense and retransmit the data. A TDMA schedule is used and each and every node in the cluster is assigned to a transmission slot.

Hence APTEEN will be using a modified TDMA schedule for implementing the hybrid network. The main features of the APTEEN scheme will contain the following:-

It will combine both the proactive and reactive policies. It will also offer a lot of flexibility by allowing the user to set the count-time interval (CT) and the threshold values for the energy consumption can be controlled by changing the count time as well as the threshold values. The actual problem of using the scheme is that an additional complexity is required to implement the threshold functions and the count time. The simulation of TEEN and APTEEN had shown that this two protocols had outperformed LEACH. The experiments had shown that APTEENs performance is somewhere between LEACH and TEEN when expressed in terms of energy dissipation and network lifetime. TEEN always give the best performance as it will decrease the number of transmissions. The actual drawback of the two approaches are the overhead and the complexity which will associate with forming clusters at multiple levels, the method of implementing threshold based functions and the way to deal with attribute-based naming of queries.

\section{PROPOSED AlgorithM For HIERARCHY BASED ROUTING}

The approach of hierarchy based routing algorithm targets to conserve energy of the sensor networks while clustering and reduce the number of hops for data transmission between clusters.

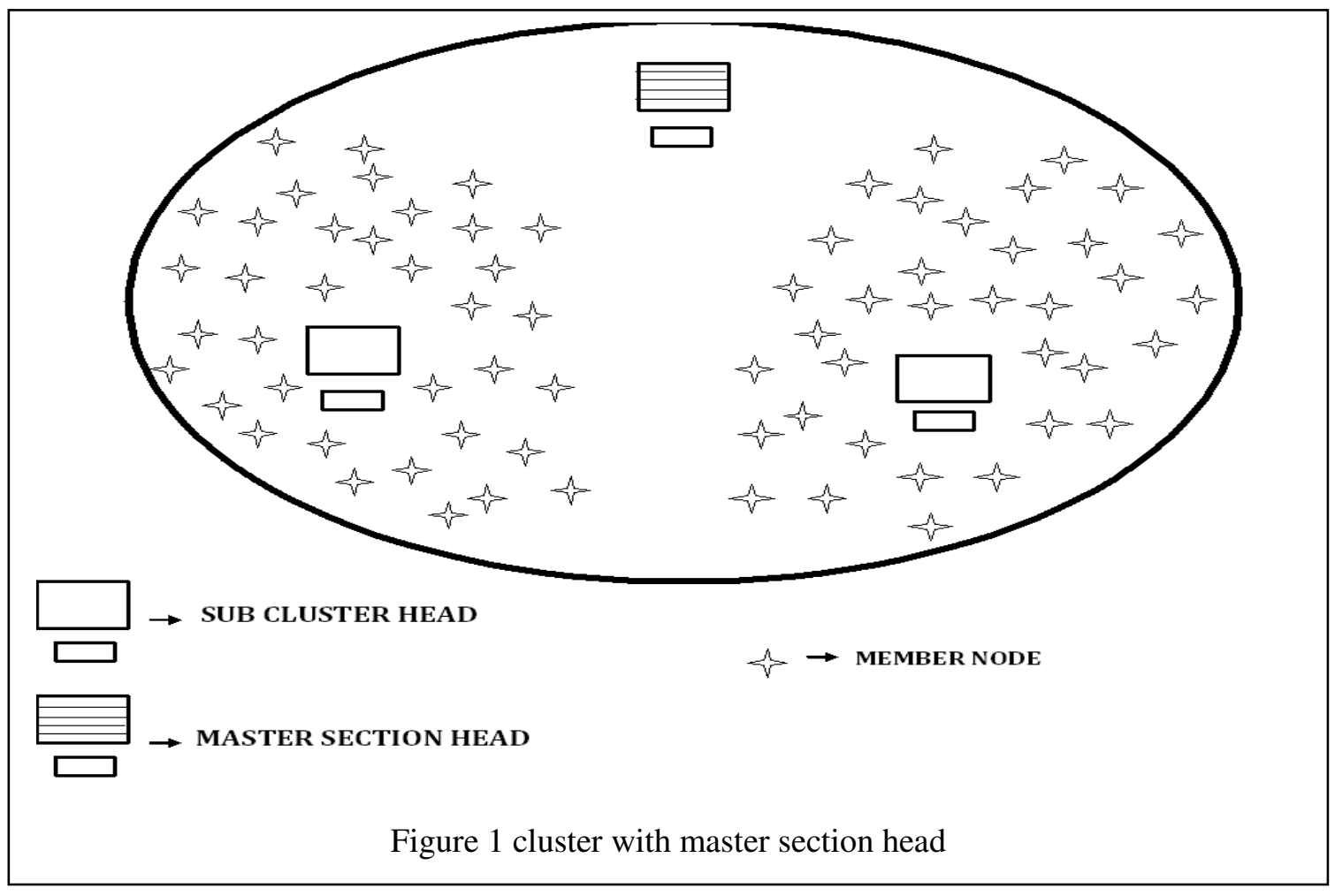


Energy is most significant parameter in cluster formation. Let the initial energy of each node is considered as a constant of 8 joules. In this algorithm, every cluster has three cluster heads and 70 nodes which are divided into two groups. Each group consist of a sub-cluster head $(\mathrm{CH})$ with 35 nodes. Thus a cluster is designed to have 2 sub cluster head and a master section head. Subcluster head gathers the data and check for event occurrence. If an event occurred then sub-cluster head transmit the event occurred data to the master section head. The master section head then transmit the data to the sink by multi-hop. More sensor nodes are used in a single cluster in predefined manner. The energy of the network is calculated after the cluster formation, size of data packet transmitted, and number of hops to reach the destination. All of them reflect the energy consumed. Energy consumption is varied depending upon the hops and the amount of data transmitted. Message communications is the number of communications occurred between any pair of nodes while clustering the network. Hence in this proposed work cluster (as portrayed in figure 1) consists of two cluster heads as Sub cluster heads and a master section head.

Energy consumption for the data transmitted is calculated for 50 bytes of data. In this proposed work, computation for the cluster head and cluster formation has been reduced. In the existing methodology, each and every round the elected cluster head have to inform to every member node, that it is the cluster head by calculating the residual energy of all the nodes. Here, in this algorithm, the cluster head election takes places only when the cluster head loses its residual energy below half of the initial energy level of $\mathrm{CH}$. Thereby the complexity of cluster head selection is much reduced. Energy consumption is varied depending upon the hops and the amount of data transmitted. The deployment of more nodes are deployed in an area will also increases the sensing capability of the network. In this algorithm number of clusters is reduced as each cluster which consists of more sensing nodes. Communication cost will also be decreased because of more nodes are used in moderate areas.

\section{SIMULATION RESULTS}

Network simulator (NS2) is used for the simulation of the proposed algorithm. NS2 is an event simulator that consists of a package of tools that simulates behaviour of networks. It helps creating network topologies, analyse and log events to understand the network behaviour. Events are queued and processed in the order of their scheduled occurrences.

Each cluster consists of 70 nodes and three heads. Nodes are distributed with two groups and each groups with a sub heads. Data transmitted is constant of about 50 bytes. Energy consumption of a group in a cluster is show in Figure 2. 


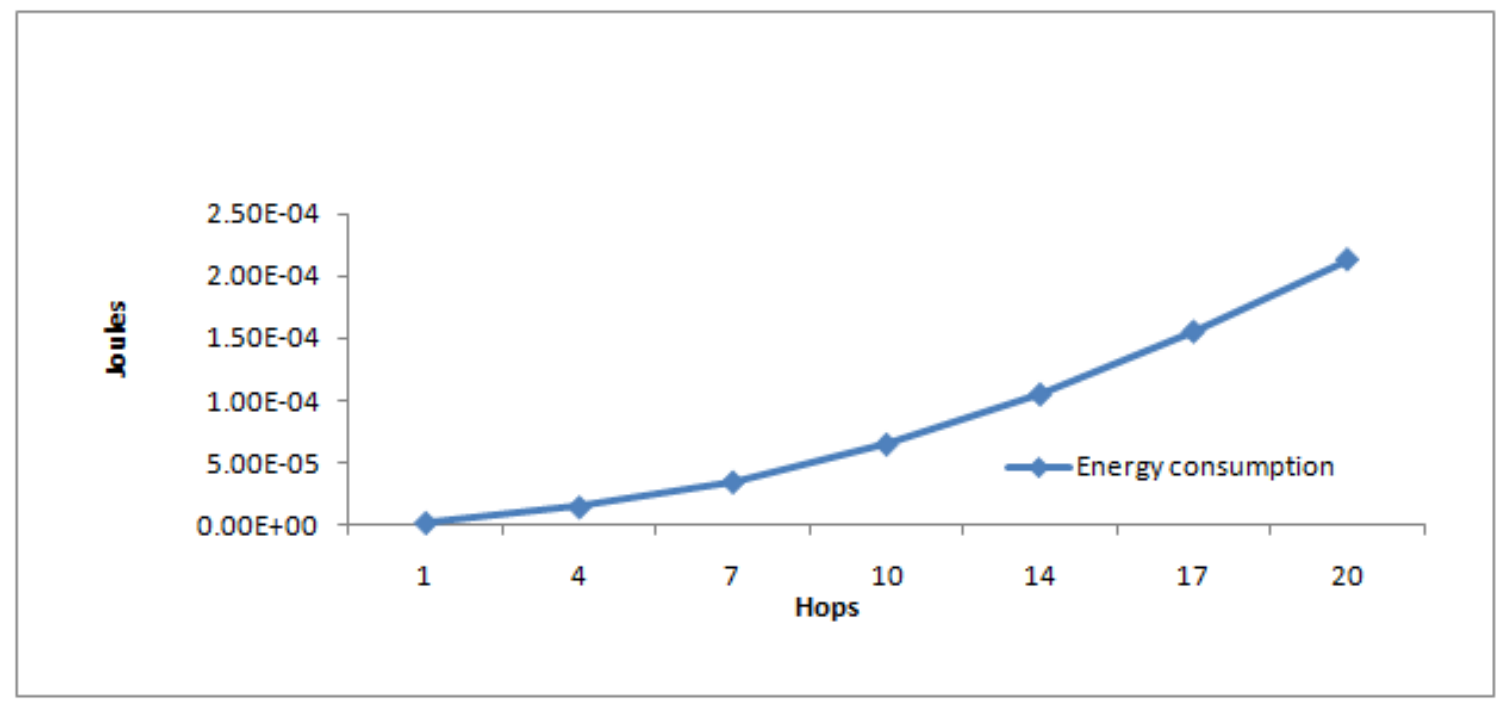

Figure 2. Energy consumption of group Vs Number of Hops

Here 50 bytes of data is transmitted for a single hop. The energy calculation is only based on the data transmitted and it doesn't include the energy consumption for routing setup phase. X-axis represents the no of hops and $\mathrm{Y}$-axis represents the energy consumption in joules. It shows the energy consumed details for different hops with a constant data of 50 bytes. The figure 3 shows, the decrease in the residual energy of the sensor nodes as more packets are received.

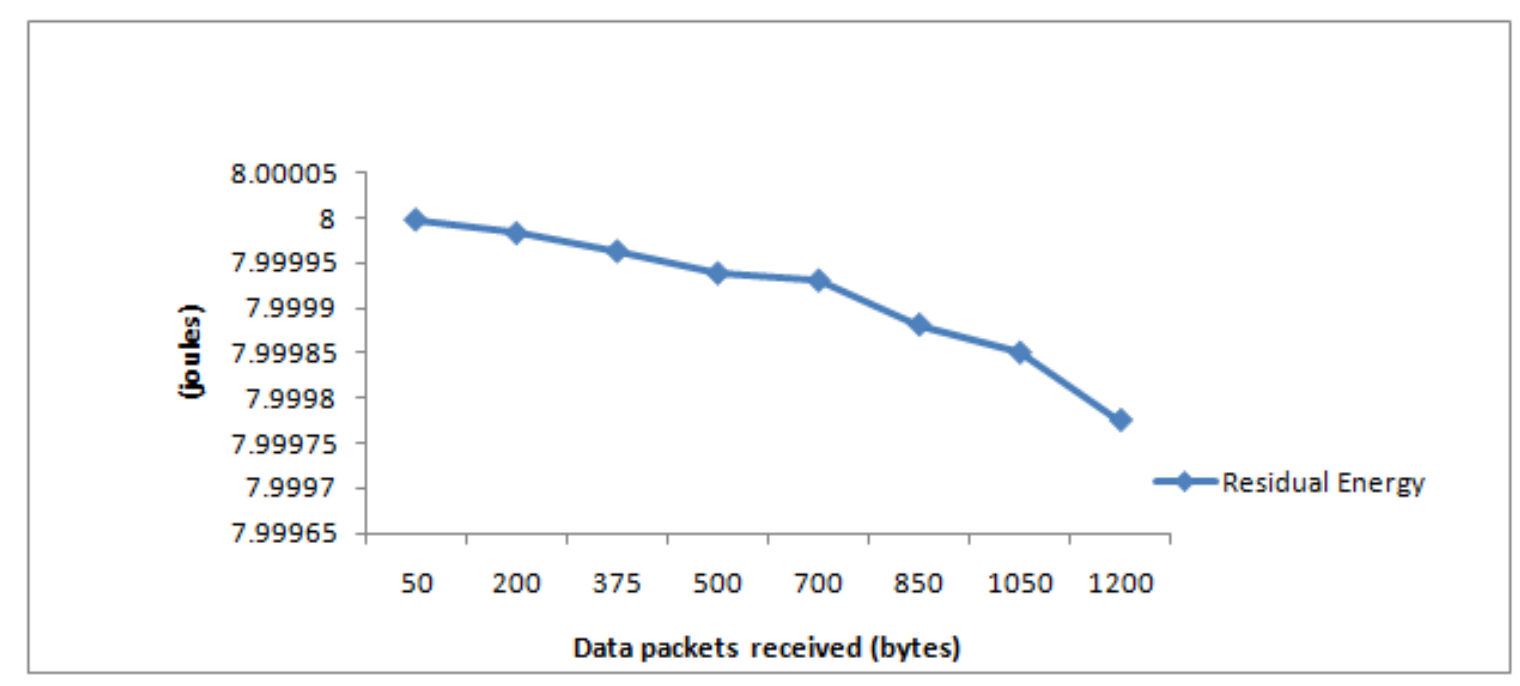

Figure 3. Residual Energy Vs Data packet received 
The $\mathrm{X}$-axis represents the no of data packets and $\mathrm{Y}$-axis represents the energy consumption in joules. As more and more data packets are received, the residual energy of the nodes reduces.

Each group consist of 35 nodes. Here the energy consumption of each group is shown. As the network size increase the corresponding energy consumption for 500 bytes of data transmission also increases. This is illustrated in figure 4.

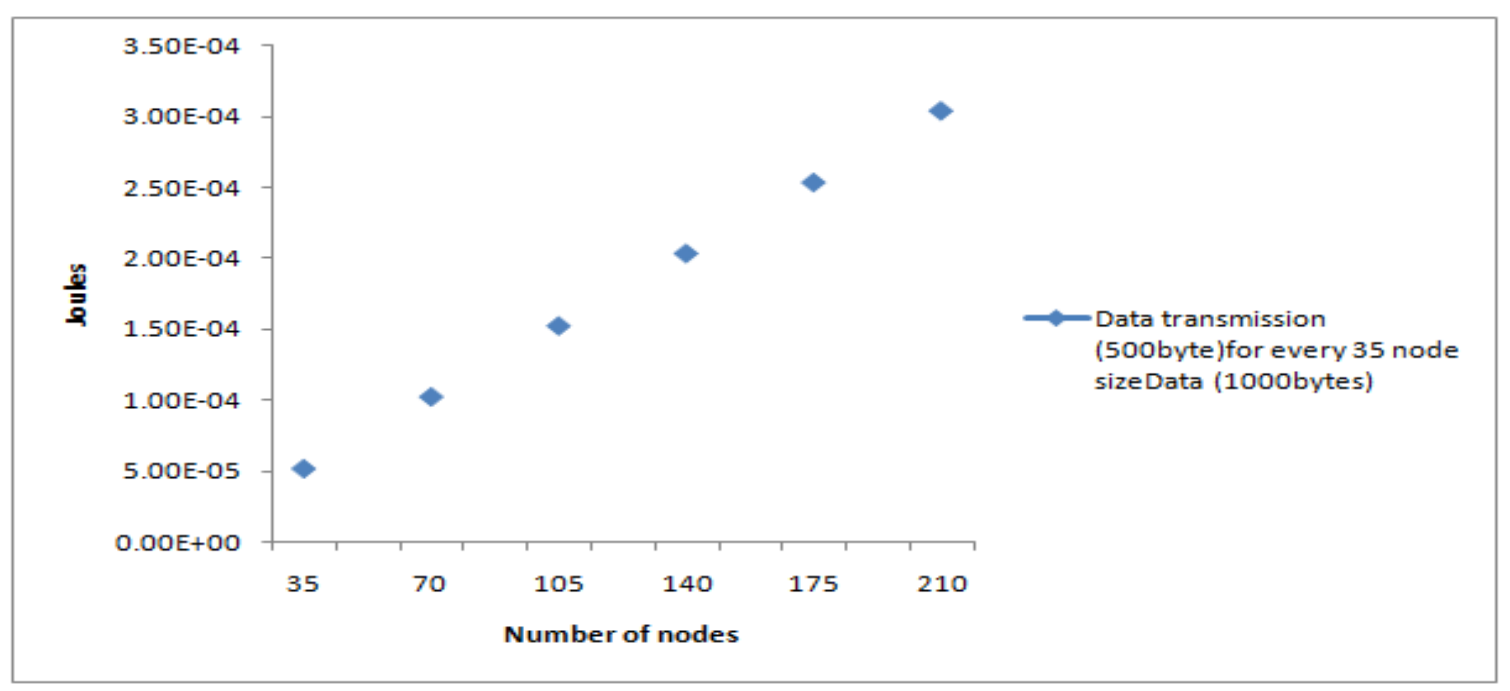

Figure 4. Energy consumption for 500 bytes of data transmitted

The $\mathrm{X}$-axis represents number of nodes and the $\mathrm{y}$-axis represents the energy consumption in joules. The energy consumption for the data transmission increases linearly with the size of the network. If the distance between the nodes increases, the energy calculated is very negligible. The increase in the network size, results in increasing the number of hops for transmission of data as shown in figure 5 .

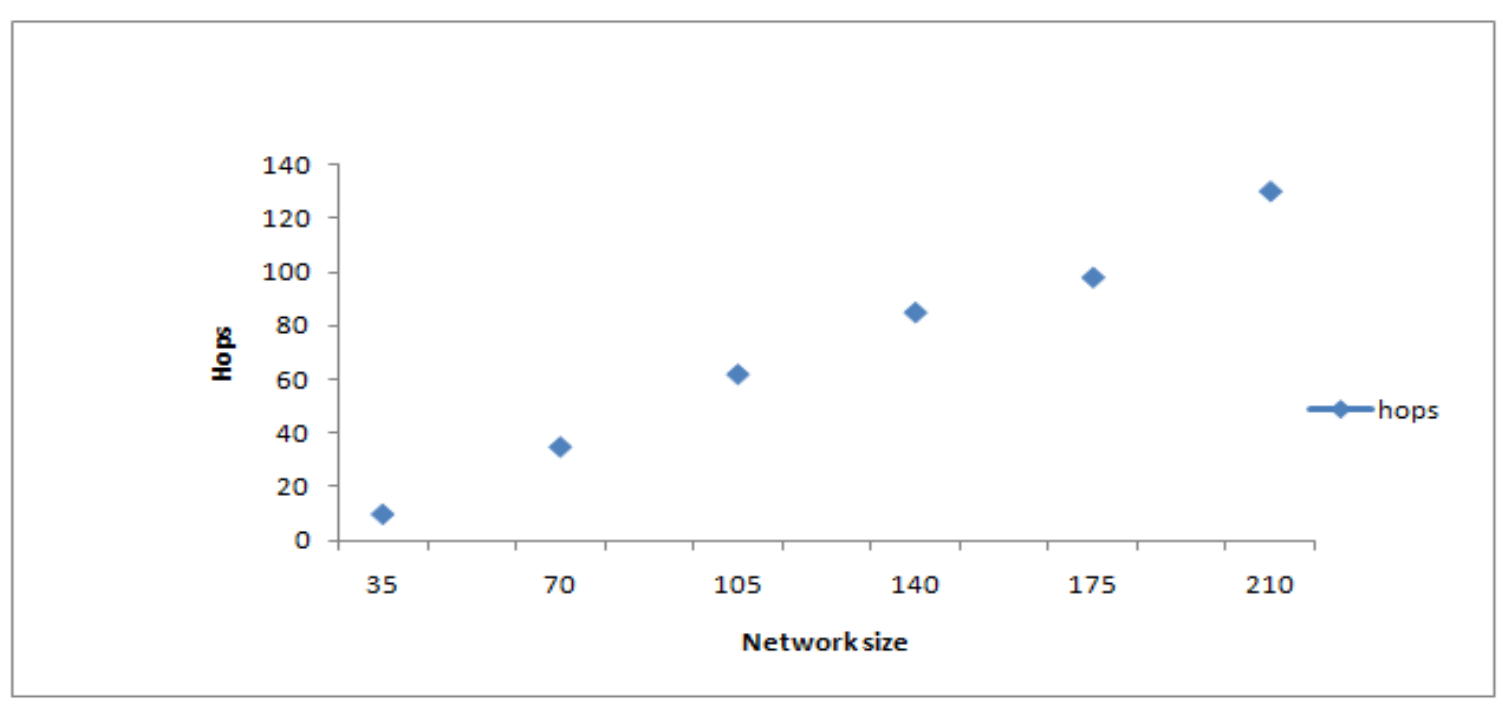

Figure 5.Network size VS Transmission Hops. 
The $\mathrm{X}$-axis represents the network size and the $\mathrm{Y}$-axis represents the no of hops. The increase in the network size will have an linear impact in the no of hops of data transmission.

\section{CONCLUSION}

The energy efficient hierarchy based protocols, proposed in this paper has resulted in a significant improvement in the performance of the network over a centralised approach. As a result of the algorithm proposed, where the initial energy of the nodes and cluster head are defined, the energy consumed for the computation is less as shown in the simulation results. Based on the cluster head election proposed in this paper, the complexity of the network and its energy consumption is much reduced. The nodes being deployed in large numbers within moderate area, demonstrates the reduction in communication cost.

\section{FUTURE WORK}

This algorithm will be extended to use mobile agent for data transmission, which works to gather and transmit the data in an efficient manner. This reduces the complexity and the Latency.

\section{REFERENCE}

[1] Y. Xu, J. Heinemann and D. Estrin. Geography-informed energy conservation for ad hoc routing. In Proceedings of the Seventh Annual International Conference on Mobile Computing and Networking (MobiCom), 2001.

[2] Boulis A, Ganeriwal S and Srivastava M B," Aggregation in sensor networks: an Energy-Accuracy Trade-Off," Elsevier journal of Ad Hoc Networks, 2003, 1(1), pp.317-331.

[3] C. Intanagonwiwat, R. Govindan and Estrin D. Directed diffusion: A scalable and robust communication paradigm for sensor networks. In Proceedings of the Sixth Annual International Conference on Mobile Computing and Networks (MobiCom), 2000.

[4] 'Handbook of Sensor Networks: Compact Wireless and Wired sensing systems' by Mohammad Ilyas and Imad Mahgoub. CRC press.[5] J. Elson and D. Estrin. "An Address-Free Architecture for Dynamic Sensor Networks". Technical Report 00-724, Computer Science Department, USC, January 2000.

[6] T. Rappaport, Wireless Communications: Principles \&Practice.Englewood Cliffs,NJ: Prentice- Hall, 1996.

[7] W. B. Heinzelman. "Application-Specific Protocol Architectures for Wireless Networks". PhD thesis, Massachusetts Institute of Technology, June 2000. 